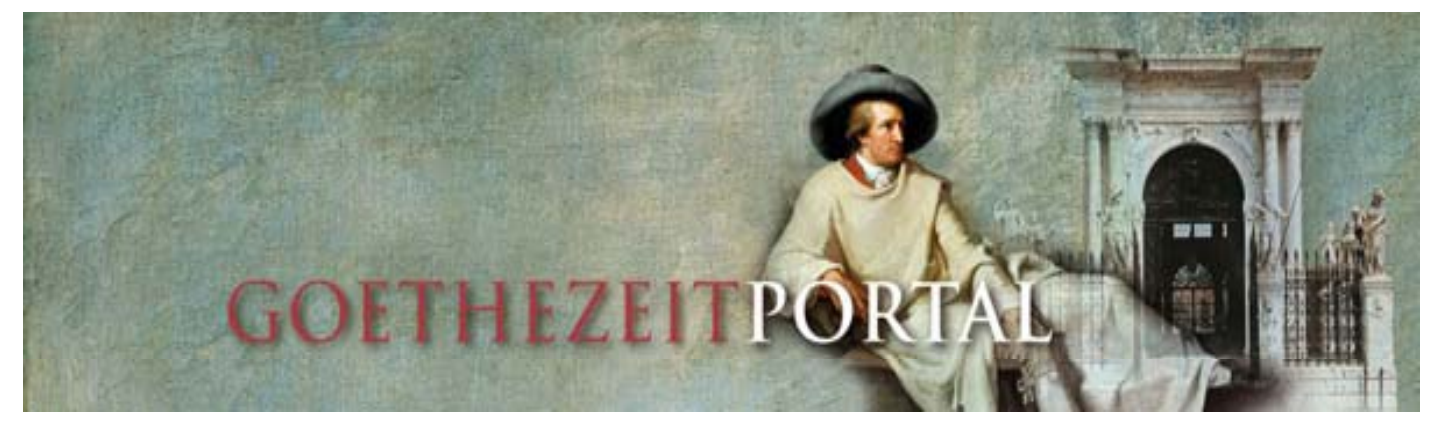

Martin Dönike

„Diese hier beygelegte Nachrichten sind mir von H. Hirt mitgetheilt worden" - Anmerkungen zur ersten Beschreibung von Tischbeins Gemälde „Goethe in der Campagna di Roma“

\title{
Erstpublikation:
}

Goethe-Jahrbuch 118 (2001), S. 354-359.

Vorlage:

PDF-Datei des Autors.

URL: <http://www.goethezeitportal.de/db/wiss/tischbein/doenike_goethe_campagna.pdf > Eingestellt am 10. Januar 2008

\author{
Autor: \\ Dr. Martin Dönike \\ Sonderforschungsbereich 644 „Transformationen der Antike“ \\ Humboldt-Universität zu Berlin \\ Unter den Linden 6 \\ 10099 Berlin \\ E-Mail: <martin.doenike@staff.hu-berlin.de>
}


MARTIN DÖNIKE

\section{„Diese hier beygelegte Nachrichten sind mir von H. Hirt mitgetheilt worden”- Anmerkungen zur ersten Beschreibung von Tischbeins Gemälde „Goethe in der Campagna di Roma”}

Johann Heinrich Wilhelm Tischbeins Gemälde Goethe in der Campagna di Roma (1786/87) ist sicherlich eines der bekanntesten Porträts des Dichters und zugleich - nicht zuletzt durch die Bearbeitung Andy Warhols - eines der berühmtesten Dichterbildnisse überhaupt. Von der Nachwelt als Sinnbild der Italienreise Goethes verstanden, verbindet sich mit ihm der Gedanke einer Synthese von antikem und modernem Kunstschaffen, die von Tischbein in der Gestalt des auf den Überresten des Altertums ruhenden Dichters der Iphigenie vor Augen geführt wird. ${ }^{1}$

Neben den entsprechenden Briefen Goethes und Tischbeins ${ }^{2}$ wird im Hinblick auf Entstehung und Deutung des Gemäldes stets auf eine im März 1788 im Teutschen Merkur erschienene und auf den 30. August 1787 datierte Beschreibung des Bildes verwiesen, die als die erste öffentliche Nachricht und, wiewohl ungezeichnet, genaueste schriftliche Quelle über das damals noch nicht vollendete Werk gelten kann. ${ }^{3}$

Vorlage für diesen unter der Überschrift Auszüge aus Briefen von Rom erschienenen Text ist ein heute im Düsseldorfer Goethe-Museum (Anton-und-Katharina-Kippenberg-Stiftung, Signatur KK 4645) aufbewahrtes anonymes Manuskript, das - anders als die Druckfassung - das Datum des 30. Juni 1787 trägt. ${ }^{4}$ Seit Karl Wagners Edition der Briefe aus dem Freundeskreise von Goethe, Herder, Höpfner und Merck (Leipzig 1847) wird das Düsseldorfer Manuskript als ein an Johann

\footnotetext{
${ }^{1} \mathrm{Zu}$ Tischbeins Gemälde siehe u.a.: Die Bildnisse Goethes. Hrsg. von Ernst Schulte-Strathaus. München 1910, S. 32-36; Christian Beutler: J. H. W. Tischbein. Goethe in der Campagna. Eine Einführung. Stuttgart 1962; Christian Lenz: Tischbein. Goethe in der Campagna. Frankfurt/M. 1979; Petra Maisak: „Wir passen zusammen als hätten wir zusammengelebt." Goethe und Tischbein in Italien. In: J. H. W. Tischbein.. Goethes Maler und Freund. Hrsg. von Hermann Mildenberger. Schleswig 1986, S. 17-50; hier S. 33-39; Péter Ujvári: Wilhelm Tischbein: Goethe in der Campagna. In: Acta Historiae Artium. Academia Scientiarum Hungaricae 36 (1993), S. 95-132; Roland Kanz: Dichter und Denker im Porträt. Spurengänge zur deutschen Porträtkultur des 18. Jahrhunderts. München 1993, S. 172-196; [Kat.] Wiederholte Spiegelungen. Weimarer Klassik 1759-1832. Ständige Ausstellung des GoetheNationalmuseums. Hrsg. von Gerhard Schuster u. Caroline Gille. 2 Bde. München, Wien 1999, Bd. 1, S. 332 f.; [Kat.] Mehr Licht. Europa um 1770. Die bildende Kunst der Aufklärung. Hrsg. von Herbert Beck, Peter Bol u. Maraike Bückling. München 1999, Kat. Nr. 12, S. 31 f.

${ }^{2}$ Zusammengestellt bei Beutler (Anm. 1), S. 22-30 und Lenz (Anm. 1), S. 9-12.

${ }^{3}$ Auszüge aus Briefen von Rom. Rom den 30. Aug. 1787. In: Der Teutsche Merkur vom Jahre 1788. Hrsg. von Chr[ristoph] M[artin] Wieland. Weimar 1786, 1. Vierteljahr, März, S. 266-272; hier S. 270-71. Neben dem Porträt Goethes beschreibt der Verfasser hier ebenfalls die Urfassung von Tischbeins heute im Landesmuseum Oldenburg befindlichen Gemälde Des Mannes Stärke.

${ }^{4}$ Siehe den Katalog der Sammlung Kippenberg. Zweite Ausgabe. 2 Bde. u. ein Registerbd. Bd. 2. Leipzig 1928, S. 73; [Ausst. Kat.] Veränderungen 1774:1794. Goethe, Jacobi und der Kreis von Münster. Hrsg. von Jörn Göres. Düsseldorf 1974, S. 125, Nr. 184. - Der gesamte Text abgedr. in: Briefe aus dem Freundeskreise von Goethe, Herder, Höpfner und Merck. Eine selbständige Folge der beiden in den Jahren 1835 und 1838 erschienenen Merckischen Briefsammlungen. Hrsg. von Karl Wagner. Leipzig 1847, S. 271-274. Einen Vergleich des Ms. mit den von Merck überarbeiteten Passagen bietet Ujvári (Anm. 1), S. $100 \mathrm{f}$.
} 
Heinrich Merck gerichteter Brief angesehen und als solcher einem Vetter Tischbeins, dem Maler Ludwig Philipp Strack, zugeschrieben. Anders als seine Nachfolger war sich Wagner des hypothetischen Charakters seiner Zuschreibung noch bewußt. In einer Anmerkung zu dem von ihm transkribierten Text heißt es: „Dem Briefe fehlt die Unterschrift, vielleicht auch der Schluß. Ich habe darum den Namen des Briefschreibers nach Vermutung angegeben, dazu veranlaßt durch die Nachschrift Strack's zu dem letzten Brief in den Briefen an Merck S. 514 und die Aehnlichkeit beider Handschriften". 5

Mit der Zuschreibung des Textes an Ludwig Philipp Strack (1761-1836) steht jedoch der Umstand in Konflikt, daß dieser erst im Jahre 1789 nach Rom gelangte, nachdem ihm kurz zuvor ein dreijähriges Reisestipendium der Kasseler Kunstakademie zugesprochen worden war. ${ }^{6}$ Folgt man diesen frühesten Quellen sowie den von Hermann Knackfuß mit gleichem Ergebnis ausgewerteten Akten der Kasseler Kunstakademie, so kann Strack das Bild im Jahre 1787 also gar nicht gesehen, geschweige denn beschrieben haben.

In der Tat ist bzw. wäre der von Wagner Strack zugeschriebene „Brief”, dem seltsamerweise nicht nur die Unterschrift, sondern auch die Anrede fehlt, das einzige Zeugnis für einen früheren Reiseantritt bzw. für einen etwaigen ersten Italienaufenthalt des Malers, der in das Jahr 1787 fallen müßte. Während Thieme-Beckers Allgemeines Lexikon der bildenden Künstler (1938) mit Hinweis auf den Brief an Merck die erste Variante vertritt, dabei jedoch den frühen Quellen widersprechen muß, ${ }^{7}$ ist neuerdings, im Katalog der Ausstellung Sehnsucht nach dem Süden. Oldenburger Maler sehen Italien (Oldenburg 2000), die zweite Variante als Lösung des biographischen

\footnotetext{
${ }^{5}$ Wagner 1847 (Anm. 4), S. 271. Bei dieser „Nachschrift” handelt es sich um einen Zusatz Stracks zu einem Brief Tischbeins aus Neapel an Merck vom 29. Juni 1790, in dem dieser berichtet, dass sein Vetter Strack ,jetzt hier” sei und „,recht fleißig” male. Strack selbst läßt Merck wissen, „,aß mir Italien über alle Maßen wohlgefällt, und ich werde mir einmal die Freyheit nehmen, Ihnen eine kleine Beschreibung der interessantesten Gegenden zu machen" (Briefe an Johann Heinrich Merck von Göthe, Wieland und anderen Zeitgenossen. Hrsg. von Karl Wagner. Darmstadt 1835, S. 514). Daß diese Ankündigung einer „,kleinen Beschreibung” - zumal der ,interessantesten Gegenden” und nicht Kunstwerke! - sich nicht auf das Schreiben vom 30. Juni 1787 beziehen kann, hat Wagner offenbar übersehen.

${ }^{6}$ Siehe Oldenburgische Blätter 39 (1819), Sp. 615 f.; Neuer Nekrolog der Deutschen 14 (1836). 1. Teil. Weimar 1838, S. 86-92; hier S. 88 f.; Fr. Müller: Die Künstler aller Zeiten und Völker. Bd. 3. Stuttgart 1864, S. 615 f.; Allgemeine Deutsche Biographie. Auf Veranlassung [...] Seiner Majestät des Königs von Bayern Maximilian II. hrsg. durch die Historische Commission bei der Königlichen Akademie der Wissenschaften. Bd. 36. Leipzig 1893, S. 486; Allgemeines Künstler-Lexikon. Leben und Werke der berühmtesten bildenden Künstler. Hrsg. von Hans Wolfgang Singer. Bd. 4. Frankfurt/M. ${ }^{3} 1901$, S. 352. Vgl. auch Hermann Knackfuß: Geschichte der Königlichen Kunstakademie zu Kassel. Aus den Akten der Akademie zusammengestellt. Kassel 1908, S. 83. Während die Allgemeine Deutsche Biographie und der Nekrolog das Jahr 1788 für den Zuschlag des alle drei Jahre ausgeschriebenen Reisestipendiums nennen, wurde dieses Knackfuß zufolge ,nach Bericht und auf Vorschlag der Akademie" sogar erst im Mai 1789 von Landgraf Wilhelm IX. an Strack sowie den Miniaturmaler Konrad Westermayer (1765-1834) verliehen. Alle genannten Quellen stimmen jedoch darin überein, daß Strack erst ,,mit dem Frühjahr 1789" nach Rom aufbrach.

7 „1787 (nicht 1789, wie häufig angegeben [Brief S.s vom 30.6.1787 aus Rom an Joh. Heinr. Merck über das Tischbeinsche Goethebildnis]) brach er mit dem Landschafter Fr. Chr. Reinermann nach Rom auf' (Thieme-Becker: Allgemeines Lexikon der bildenden Künstler von der Antike bis zur Gegenwart. Bd. 32. Leipzig 1938, S. 144).
} 
Dilemmas vorgeschlagen worden, wobei sich allerdings keine weiteren Belege für einen ersten Italienaufenthalt Stracks finden lassen. ${ }^{8}$

Es ist indes fraglich, ob es solcher gewundener und dabei wenig überzeugender Konstruktionen bedarf, um den Widerspruch zwischen dem Düsseldorfer Manuskript als Zeugnis für Stracks Anwesenheit im Rom des Jahres 1787 und seiner von den Quellen erst für das Jahr 1789 belegten Ankunft aufzulösen. Mehrere Hinweise deuten darauf, daß Manuskript und Druckfassung der im Teutschen Merkur erschienenen Beschreibung des Tischbeinschen Gemäldes gar nicht von Ludwig Philipp Strack, sondern tatsächlich von dem damaligen Cicerone und späteren Professor an der Berliner Universität Aloys Hirt (1759-1837) stammen, der unter den kunstinteressierten und -gelehrten Deutschen, die sich in den späten 1780er Jahren in Rom aufhielten, eine besondere Stellung einnahm. ${ }^{9}$ Es war Hirt, der sowohl Goethe als auch Herder durch Rom führte und der in den Jahren 1789 bis 1791 gemeinsam mit Karl Philipp Moritz die Zeitschrift Italien und Deutschland herausgeben sollte, in der sich mehrere Aufsätze von seiner Hand über zeitgenössische Künstler wie z.B. Drouais, Dannecker, Scheffauer und Trippel finden. Hirt lebte bereits seit 1782 in Italien. Er kehrte erst 1796 nach Deutschland zurück und hielt sich also im Jahre 1787 nach wie vor in Rom auf, wo er neben Goethe auch mit dem anderen Protagonisten der Beschreibung, dem Maler Tischbein, verkehrte. ${ }^{10}$

Wichtigster Beleg für die hier vertretene These, daß Hirt der Verfasser der berühmten Gemäldebeschreibung ist, ist ein Brief, den Johann Heinrich Merck an den damaligen Herausgeber des Teutschen Merkur, Christoph Martin Wieland, richtete. Unter den zahlreichen Personen, mit denen Wieland korrespondierte, kommt Merck insofern eine besondere Bedeutung zu, als er neben eigenen Beiträgen immer wieder auch Arbeiten anderer Verfasser an seinen „lieben Herrn Bruder" sandte, die dieser, sofern möglich, in den Merkur einrücken sollte. Vom 19. Februar 1788 nun ist ein Brief Mercks überliefert, in dem er Wieland wissen läßt, daß die seinem Schreiben ,,beygelegte[n] Nachrichten” ihm „,von H. Hirt mitgetheilt worden” seien und Tischbein „,zugleich gebeten [habe], daß man seiner in einem Deutschen Journal wieder Einmal gedächte”. Er selbst, so Merck weiter, habe „einige kleine Schnizzen hinzugethan, um meinem würdigen Freunde, dem Sohne des berühmten Camper ein kleines Denkmal zu sezen, das nichts der Wahr-

\footnotetext{
${ }^{8}$ Siehe den Beitrag zu Stracks Leben und Werk von Silke Liesenfeld im Ausstellungskatalog Sehnsucht nach dem Süden. Oldenburger Maler sehen Italien. Sonderausstellung des Landesmuseums im Oldenburger Schloß vom 5. Mai bis 19. November 2000. Oldenburg 2000, S. 155 f. u. S. 183 f. (Zeittafel).

${ }^{9}$ Siehe den Brief Maler Müllers an Heinse, 17.4.1787; in: Friedrich Müller, genannt Maler Müller, Briefwechsel. Kritische Ausgabe. Hrsg. von Rolf Paulus u. Gerhard Sauder. Teil 1: Briefwechsel 1773-1811. Heidelberg 1998, S. 119.

${ }^{10} \mathrm{Zu}$ Hirt siehe den Eintrag von Wolfgang Frhr. von Löhneysen in: Neue Deutsche Biographie. Bd. 9. Berlin 1972, S. 234 f. (mit weiterer Literatur) sowie Jürgen Zimmer: Nachrichten über Aloys Hirt und Bibliographie seiner gedruckten Schriften. In: Jb. der Berliner Museen N. F. 41 (1999), S. 133-194. Zu Hirts römischer Zeit siehe u.a. Julius Vogel: Aus Goethes Römischen Tagen. Kultur- und kunstgeschichtliche Studien zur Lebensgeschichte des Dichters. Leipzig 1905, S. 243 ff.; Friedrich Noack: Deutsches Leben in Rom 1700-1900. Stuttgart, Berlin 1907, S. 119 u. passim.
} 
heit entgegengesetztes” enthalte, und fügt an: „Mir geschieht ein grosser Dienst, wenn Dieses Alles in irgend einer Ecke des Vacui des Deutschen Merkur Plaz finden kann; u. zwar bald". ${ }^{11}$

$\mathrm{Da}$ Hirt sich mit seinem Manuskript an Merck wandte, überrascht nicht, hatte dieser doch schon zuvor einige Arbeiten Hirts, mit dem er spätestens seit 1785 in Kontakt stand, an Wieland vermittelt. ${ }^{12}$ Zeugnis dafür sind drei im Teutschen Merkur erschienene „Briefe aus Rom”, in denen Hirt bereits 1785/86 über die neuesten Werke von Angelika Kaufmann, Albrecht Christoph Dies und Jacques-Louis David sowie - nicht zuletzt - über Tischbeins ,in Rom verfertigtes Gemählde" Konradin von Schwaben berichtet hatte. ${ }^{13}$

Bekanntlich hat auch Goethe in einem Brief an Wieland vom 17. November 1786 Hirt als Mitarbeiter für den Teutschen Merkur empfohlen. Außer über antike Kunstwerke, den römischen Kunsthandel und die dortigen Kunstsammlungen könne Hirt, so schlug er Wieland vor, u. a. auch von „Wercken lebender Künstler die theils in Rom seßhaft sind theils, sich daselbst eine Zeitlang aufhalten, in allen Theilen der Kunst" berichten. ${ }^{14}$

Auch wenn Wieland Goethes „Anerbieten”, Hirt zu einem festen Mitarbeiter des Merkur zu machen, ablehnte, ${ }^{15}$ bedeutete dies offenbar nicht, daß er Beiträge Hirts generell abzulehnen gedachte: So entschied er sich im Falle der von Merck übersandten „Nachrichten”, diese in den Merkur aufzunehmen. Schon im Märzheft der Zeitschrift erschien die mit dem Datum des 30. August 1787 versehene Beschreibung der neuesten Arbeiten Tischbeins sowie, in der fingierten Form eines auf den 30. Dezember 1787 datierten Briefes, die von Merck ebenfalls erwähnten eigenen „Schnizzen”, beide unter der Rubrik Auszüge aus Briefen von Rom und ohne Nennung der Verfassernamen.

Offenbar allein aufgrund der zeitlichen Koinzidenz von Mercks Schreiben an Wieland vom 19. Februar und der Publikation der beiden „Auszüge aus Briefen von Rom” im Märzheft des Merkur haben bereits Herbert Kraft wie auch Thomas C. Starnes und Uta Motschmann stillschweigend Hirt als Verfasser der Beschreibung von Tischbeins Gemälde angenommen, ohne jedoch auf das in Düsseldorf aufbewahrte Manuskript einzugehen, das von der literatur- und kunstgeschichtlichen Forschung nach wie vor Strack zugeschrieben wird. ${ }^{16}$ Dabei lassen sich

\footnotetext{
11 Merck an Wieland, 19.2.1788; zit. nach: Wielands Briefwechsel Hrsg. von der Berlin-Brandenburgischen Akademie der Wissenschaften durch Siegfried Scheibe. Bd. 9/1 (bearbeitet von Uta Motschmann). Berlin 1996, S. 399.

${ }^{12}$ Siehe das Antwortschreiben Wielands an Merck, 5.12.1785; in: Wielands Briefwechsel(Anm. 11), S. 101.

${ }^{13}$ Briefe aus Rom, hauptsächlich neue Werke jetzt daselbst lebender Künsder betreffend [1-3]. In: Der Teutsche Merkur vom Jahre 1785. Hrsg. von Chr[istoph] M[artin] Wieland. Weimar 1785, 4. Vj., Dezember, S. 251-267; Der Teutsche Merkur vom Jahre 1786. 1. Vj., Januar, S. 69-82; Der Teutsche Merkur vom Jahre 1786. 1. Vj., Februar, S. 169-186. Vgl. dazu Zimmer (Anm. 10), S. 168 f.

${ }^{14}$ Wielands Briefwechsel (Anm. 11), S. $195 \mathrm{f}$

${ }^{15}$ Wieland an Merck, 17.12.1786; zit. nach: Wielands Briefwechsel (Anm. 11), S. 210.

${ }^{16}$ Johann Heinrich Merck. Briefe. Hrsg. von Herbert Kraft. Frankfurt/M. 1968, S. 796; Thomas C. Starnes: Christoph Martin Wieland. Leben und Werk. 3 Bde. Bd. 2: „Der berühmteste Mann in Deutschland” 1784-1799. Sigmaringen 1987, S. 128,
} 
Dönike: „Diese hier beygelegte Nachrichten“, S. 5

mehrere Argumente dafür anfuhren, daß das dem gedruckten Text zugrundeliegende Manuskript in der Tat von Aloys Hirt stammt.

Hält man das Düsseldorfer Manuskript neben das von Hirt wohl im selben Jahr für Goethe zusammengestellte Verzeichniß der bekanntesten jetztlebenden Künstler in Rom, so ist die Ähnlichkeit der Handschriften unverkennbar; sie ist nicht nur auf das allgemeine Erscheinungsbild beschränkt, sondern läßt sich auch an der graphisch nahezu identischen Schreibung zahlreicher Wörter festmachen. Gleiches gilt für einen nur etwa ein Jahr später verfaßten Brief Hirts an Goethe vom 23. August $1788 .{ }^{17}$ Doch nicht nur paläographische, auch inhaltliche bzw. stilistische Gründe sprechen dafür, in Hirt den Verfasser der Nachricht über Tischbeins neueste Arbeiten zu sehen. Neben der an Winckelmanns Geschichte der Kunst orientierten, durchaus gelehrten Beschreibung der auf dem Bild versammelten Antiken legt dies insbesondere der wiederholte Gebrauch des Wortes „karakteristisch” nahe, das zu den für den „Charakteristiker” Hirt typischen Vokabeln gehört. ${ }^{18}$ Der Begriff des „Karakteristischen” sollte zwar erst fast zehn Jahre später durch Hirts in Schillers Horen publizierte Aufsätze über das Kunstschöne und den Laokoon berühmt werden. Daß Hirt diesen Begriff gleichwohl bereits Ende der 1780er Jahre im Mund geführt hat, belegt ein Brief von seiner Hand an Goethe vom April 1789, in dem er davon spricht, daß „Bedeutung, Karakteristik, Wahrheit” das „erste Grundgesez der bildenden Künste” seien. $^{19}$

Schließlich fällt der Name Hirts in dem Text Mercks, der direkt auf den Brief über die neuesten Arbeiten Tischbeins folgt und wie dieser unter die Rubrik Auszüge aus Briefen von Rom

Fußnote 11; Wielands Briefwechsel (Anm. 11), S. 490 f. Vgl. neuerdings auch Norbert Christian Wolf: Streitbare Ästhetik. Goethes kunst- und literaturtheoretische Schriften 1771-1789. Tübingen 2001, S. 263 u. S. 522. Dagegen führt Zimmer (Anm. 10) diesen Text in seiner Bibliographie der gedruckten Schriften Hirts nicht an, womit er einem Schriftenverzeichnis Hirts aus dem Jahre 1826 folgt (Verzeichniss im Jahre 1825 in Berlin lebender Schriftsteller und ihrer Werke. Aus den von ihnen selbstt entworfenen oder revidierten Artikeln zusammengestellt und zu einem milden Zwecke herausgegeben. Berlin 1826, S. 107-109). Die Tatsache, daß sich der Bericht über Tischbeins Goethe-Bild in diesem Verzeichnis nicht findet, darf jedoch nicht überbewertet werden, da die Liste zumindest im Bereich der Zeitschriftenaufsätze unvollständig ist. So findet sich zum Beispiel kein Hinweis auf Hirts Aufsatz Das Bildniß von Göthe, gemalt von F. Büry, erschienen in: Berlinisches Archiv der Zeit und ihres Geschmacks. September 1800, S. 232-241.

${ }^{17}$ Das undatierte handschriftliche Verzeichniß Hirts hat Johann Heinrich Meyer in seinem Nachlaß aufbewahrt (Goethe- und Schiller-Archiv Weimar, Signatur 64/111,1). Der Text von Hirts Brief an Goethe vom 23. August 1788 (Goethe- und Schiller-Archiv Weimar, Signatur 28/1041, S. 175-178) findet sich abgedr. in Harnack (Anm. 19), S. 52-54. Man vergleiche z. B. die Schreibung der in beiden Texten vorkommenden Worte „Attitude” und „Künstler”. Frühere Briefe von der Hand Hirts scheinen nicht überliefert zu sein.

18 „Tischbein hatte also alle Muße, die Züge und den Charakter seines Gastfreundes zu studiren”; zit. nach der orthographisch zum Teil korrigierten Transkription von Wagner 1847 (Anm. 4), S. 273. Vgl. ebd.: „Der Künstler hat sich bemüht die Ähnlichkeit und die charakteristischen Züge seines Urbildes so viel [wie] möglich zu treffen” (ebd., S. 274). - Schon zuvor, im Abschnitt über das Bild Des Mannes Stärke, hatte es geheißen: „Man sieht zwey solcher Natur-Menschen, deren Gestalt derjenigen charakteristischen der Helden der Alten gleichkömmt” (ebd., S. 272).

${ }^{19}$ Hirt an Goethe, 4.4.1789; zit. nach: Otto Harnack (Hrsg.): Zur Nachgeschichte der italienischen Reise. Goethes Briefwechsel mit Freunden und Kunstgenossen in Italien 1788-1790. Weimar 1890, S. 163. - Zu Hirts Lehre von der Charakteristik siehe neuerdings: Alessandro Costazza: Das „,Charakteristische” als ästhetische Kategorie der deutschen Klassik. Eine Diskussion zwischen Hirt, Fernow und Goethe nach 200 Jahren. In: Jb. der Deutschen Schillergesellschaft 42 (1998), S. 64-94 (dort auch eine kritische Zusammenstellung der älteren Literatur). 
gehört. ${ }^{20}$ Bei diesem Text handelt es sich, wie gesagt, um die von Merck in seinem oben zitierten Schreiben an Wieland erwähnten „Schnizzen”, mit denen er ,dem Sohne des berühmten Camper ein kleines Denkmal" setzen wollte. Auch wenn Merck selbst nie in Rom war, so enthält sein fiktiver Brief doch „nichts”, wie er schreibt, „der Wahrheit entgegengeseztes”, insofern Adrian Gilles Camper, Sohn des mit Merck befreundeten Anatomen Petrus Camper, sich 1788 tatsächlich in Rom aufgehalten hatte und dort u.a. mit Goethe zusammengetroffen war. Dem Briefbericht über das Zusammentreffen mit dem jungen Camper fügte Merck nun eine Bemerkung an, die vom Inhalt her gänzlich unmotiviert scheint, durch einen Absatz jedoch eigens hervorgehoben ist: Hinter der Maske eines fiktiven Ichs behauptet Merck hier, daß er Camper „die besondere Verbindlichkeit schuldig” sei, ihn ,mit Herrn Hirt, einem teutschen sehr geschickten Antiquar, bekannt gemacht” zu haben, den er ,,allen Fremden, besonders denen von unserer Nation, mit Nachdruck empfehlen” könne. Nicht zuletzt Goethe, „der berühmte Verfasser des Göz von Berlichingen”, habe sich ,seiner Führung anfangs [...] überlassen”. ${ }^{21}$ Die Nennung Hirts, dessen Kenntnisse als Cicerone hier unverhohlen angepriesen werden, läßt sich als verdeckter, dabei jedoch unmißverständlicher Hinweis auf Hirt als Verfasser des unmittelbar zuvor abgedruckten Briefes verstehen - als Hinweis, dem damit der Charakter einer versteckten Signatur zukommt. Neben die briefliche Äußerung Mercks an Wieland, daß die von ihm „beygelegten Nachrichten” von Hirt stammten, dem Befund der Handschrift und dem kunsthistorisch wie auch -theoretisch einschlägigen Vokabular des Textes tritt somit ein textimmanenter Beleg für die These, daß die im Teutschen Merkur veröffentlichte Nachricht über Tischbeins Gemälde Goethe in der Campagna di Roma nicht von Ludwig Philipp Strack, sondern von Aloys Hirt stammt.

Wenn Hirt die Beschreibung der Tischbeinschen Gemälde tatsächlich, wie hier vorgeschlagen, an Merck geschickt hat, um sie im Teutschen Merkur veröffentlichen zu lassen, so bedeutet dies aber auch, daß das im Düsseldorfer Goethe-Museum aufbewahrte Schreiben kein Brief im eigentlichen Sinne, sondern ein Manuskript Hirts ist, das einem an Merck gerichteten Brief beigelegt war. ${ }^{22}$ Diese Erklärung läßt auch verständlich werden, warum dem auf vier Seiten eines einfach gefalteten Bogens niedergeschriebenen Text sowohl die Anrede als auch der für einen Brief übliche Schluß samt Unterschrift fehlen. Der vermeintlich persönliche Brief ist also keinesfalls unvollständig überliefert, wie Wagner 1847 spekuliert hatte; als von Anfang an zur Veröffentlichung gedachtes fiktives Schreiben ist der Text durchaus vollständig.

\footnotetext{
${ }^{20}$ Auszüge aus Briefen von Rom (Anm. 3), S. 271 -273. Vgl. dagegen Kraft und Starnes (Anm. 16), die behaupten, daß Merck den Text nicht vollendet habe.

${ }^{21}$ Ebd., S. 272 f.

${ }^{22}$ Allem Anschein nach handelt es sich dabei um den Brief Tischbeins an Merck aus Neapel, 10.10.1787, in dem Tischbein auf die beigelegte Beschreibung zweier Bilder von ihm hinweist. Siehe Wagner 1835 (Anm. 5), S. 509 f.
} 
Die hier vorgeschlagene Neuzuschreibung der Nachricht über Tischbeins berühmtes GoethePorträt an Aloys Hirt erlaubt es nicht nur, die durch die bisherige Zuschreibung bedingten Widersprüche in der Biographie Ludwig Philipp Stracks aufzulösen, die in der Kunstgeschichte immer wieder für Verwirrung gesorgt haben. Tatsächlich dürfte Strack wohl erst im Jahre 1789 gemeinsam mit dem Landschaftsmaler Friedrich Christian Reinermann (1764-1835) nach Italien gereist und mit Tischbein erst im Jahre 1790 in Neapel zusammengetroffen sein. ${ }^{23}$ Mit der Neuzuschreibung wird zudem ein wichtiger Quellentext der Kunst- und Literaturgeschichte des Klassizismus einem Verfasser wiedergegeben, der von der Forschung lange Zeit vernachlässigt worden ist, von Goethe jedoch trotz „oftmaliger verschiedener Meinung” zeitlebens geschätzt wurde. ${ }^{24}$ In einem Brief vom 12. August 1827, in dem er sich bei Hirt für die Übersendung der letzten Bände von dessen Geschichte der Baukunst (Berlin 1827) bedankt, läßt Goethe seinen ehemaligen Cicerone wissen: „Nun erinnert mich das übersendete Werk aufs angenehmste an gemeinsamen Eintritt in das Kunstgebiet; es giebt Zeugniss von fortwährendem parallelen Handeln und Bemühen, von convergirendem und begleitendem Thun und Wirken". ${ }^{25}$ Ein Werk mit vergleichbarem Zeugnischarakter ist auch die 1787 verfaßte Beschreibung von Tischbeins berühmtem Gemälde Goethe in der Campagna di Roma, die am Anfang einer mehr als vierzigjährigen Bekanntschaft steht und zugleich, gemeinsam mit dem Verzeichni $\beta$ der bekanntesten jetztlebenden Künstlerin Rom, als der früheste handschriftlich überlieferte Text Hirts gelten muß.

\footnotetext{
${ }^{23}$ Siehe den bereits oben (Anm. 5) zit. Brief Tischbeins an Merck, 29.6.1790.

${ }^{24}$ So Goethe an Zelter, 6.2.1827 (MA 20.1, S. 964). Erst in jüngster Zeit scheint der immerhin von Hegel in seinen Vorlesungen über die Ästhetik als ,einer der größten wahrhaften Kunstkenner unserer Zeit” bezeichnete Hirt wieder das Interesse der Forschung auf sich ziehen zu können. So wurde im April 2000 unter dem Titel Studium Antiquitatis Omnigenae in der Berlin-Brandenburgischen Akademie der Wissenschaften eine von Conrad Wiedemann und Claudia Sedlarz organisierte Tagung über Aloys Hirt als „Archäologe, Historiker, Kunstkenner” veranstaltet, deren Beiträge in einem Tagungsband publiziert werden sollen. - Hirt ist auch der Ausgangspunkt einer von Vf. vorbereiteten Dissertation, die sich der von Winckelmann verurteilten „Nachahmung des Gewaltsamen” als Problem der klassizistischen Kunsttheorie und -praxis um 1800 widmet. In diesem Zusammenhang werden Hirts in den Hören veröffentlichte Aufsätze über die Laokoongruppe und andere Werke der antiken Kunst als - klassizismusinterne - Provokation begriffen, die die vor allem in den Propyläen dargelegte Kunsttheorie der Weimarischen Kunstfreunde in entscheidender Weise beeinflußt hat.

${ }^{25}$ Goethe an Hirt, 12.8.1827; zit. nach: GJb 1894, S. 80 f.
} 